\title{
THE EFFICACY OF PLASMA EXCHANGE IN COVID-19 PATIENTS WITH COMORBID DIABETES MELLITUS
}

\author{
Komal Arshad, Zill-e-Humayun, Sultan Mehmood Kamran, Ikram Khaliq, Muhammad Raza Asif, Saman Tanveer \\ Pak Emirates Military Hospital/National University of Medical Sciences (NUMS) Rawalpindi Pakistan
}

\begin{abstract}
Objective: To explore the use of therapeutic plasma exchange as adjunctive therapy in COVID-19 patients with and without diabetes mellitus.

Study Design: Prospective, observational study.

Place and Duration of Study: Pakistan Emirates Military Hospital Rawalpindi, from Jan to Feb 2021.

Methodology: A total of 90 male patients with laboratory-confirmed coronavirus infection were selected based on our inclusion criteria and their management and outcomes were recorded. The data were analyzed using SPSS-22 and Microsoft Excel.

Results: The mortality rate was lower in patients who received 1 or more sessions of plasma exchange compared to those who did not receive plasma exchange ( $7.5 \%$ vs $12 \%)$. A lower mortality rate was seen in patients without diabetes who received therapeutic plasma exchange in addition to standard therapy compared to patients who received standard therapy alone $(0 \mathrm{vs}$ $14.82 \%, p=0.112)$. In patients with diabetes, a higher mortality rate was found in the group that had received therapeutic plasma exchange in addition to standard therapy instead of standard therapy alone ( $20 \%$ vs $8.7 \%, p=0.365)$.

Conclusion: Overall our study supports the use of therapeutic plasma exchange in COVID-19 patients. However, although statistically insignificant, there appears to be a higher mortality rate in patients with diabetes who received therapeutic plasma exchange in addition to standard therapy. As such, we recommend further investigation of this aspect.
\end{abstract}

Keywords: COVID-19, Diabetes, Mortality rate therapeutic plasma exchange.

How to Cite This Article: Arshad K, Humayun Z, Kamran SM, Khaliq I, Asif MR, Tanveer S. The Efficacy of Plasma Exchange in COVID-19 Patients with Comorbid Diabetes Mellitus. Pak Armed Forces Med J 2021; 71(5): 1732-1735. doi: https://doi.org/10.51253/pafmj.v71i5.6317

\footnotetext{
This is an Open Access article distributed under the terms of the Creative Commons Attribution License (https://creativecommons.org/licenses/by-nc/4.0/), which permits unrestricted use, distribution, and reproduction in any medium provided the original work is properly cited.
}

\section{INTRODUCTION}

According to the Center for Systems Science and Engineering (CSSE) at Johns Hopkins University (JHU) and the weekly epidemiological update by the World Health Organization (WHO), the COVID-19 pandemic has claimed over 2.2 million lives worldwide till now. 1,2 As the pandemic continues to grow and affect more people than ever, and the especially vulnerable population at risk of developing severe infection and complications, including death, is the geriatric population and those with comorbidities, such as diabetes mellitus. Several mechanisms have been proposed for the higher risk in the patients with diabetes including immuno-deficiency and altered inflammatory responses such as a reduced capacity to tolerate systemic cytokines. 3,4 COVID-19 mortality in these patients has been shown to be linked not only to the cardiovascular and renal complications of diabetes but also directly to the degree of glycemic control and Body Mass Index (BMI). 5,6

Correspondence: Dr Komal Arshad, Resident Pulmonology, Dept of Pulmonology, PEMH, Rawalpindi Pakistan

Received: 17 Feb 2021; revision received: 16 Jun 2021; accepted: 13 Aug 2021
Although preventing exposure to the virus and controlling the underlying risk factors remains the best way to prevent severe illness, a number of adjunctive therapies have been tried and studied since the start of the pandemic. These include plasma exchange, Remdesivir, and Tocilizumab. ${ }^{7}$ The potential benefits of plasma exchange in the setting of well-recognized complications, such as cytokine storm and coagulopathy, have been reported by multiple studies. ${ }^{8,9}$ In one such study, it was shown to be particularly effective when used multiple times and early in the course of the infection. ${ }^{10}$ However, few studies have commented directly on the efficacy of plasma exchange in patients with diabetes.

In our study, we aim to explore Therapeutic Plasma Exchange (TPE) as an adjunctive therapy and its potential effectiveness in male patients with and without diabetes who developed Severe Acute Respiratory Syndrome coronavirus 2 (SARS-CoV-2).

\section{METHODOLOGY}

The study was prospective and single center, conducted at Pak Emirates Military Hospital (PEMH) in Rawalpindi. It was approved by the ethical review 
committee at PEMH (certificate number: A/28/EC/ 246/2021) and spanned 4 weeks, from $6^{\text {th }}$ January 2021 to 3rd February 2021.

Inclusion Criteria: Male patients, aged 40-79 years, with laboratory confirmed SARS-CoV-2 infection and severe disease at the time of admission were included in the study.

Exclusion Criteria: Patients with moderate or critical disease severity at the time of admission were not included. Patients, who had comorbids other than diabetes mellitus, including hypertension, ischemic heart disease, chronic kidney disease, obstructive lung disease, liver disease and/or malignancy, were excluded from the study.

Convenience sampling was used and a total of 121 patients met the above-mentioned criteria during the duration of the study. The study was not interventional and all patients were managed at the discretion of their treating physicians: individuals who showed signs of cytokine release storm received Therapeutic Plasma Exchange (TPE) in addition to standard therapy; the remaining subjects received standard therapy alone. As such, no special informed consent process was required for the study apart from consent for use of patient data. Patients who received adjunctive therapies other than TPE, including Tocilizumab, Remdesivir, convalescent plasma therapy and/or mesenchymal stem cell therapy, during the course of their treatment were excluded from the study. This left behind 90 patients from the original sample.

Laboratory confirmed SARS-CoV-2 was defined as COVID-19 infection confirmed by RT-PCR sampling of nasopharyngeal and oropharyngeal swabs, at the time of admission or subsequently during the first week of admission in patients with high clinical pretest probability.

Severe disease was operationally defined as COVID-19 pneumonia with evidence of hypoxemia (ie, respiratory rate $>30 /$ minute or $\mathrm{PaO} 2<80 \mathrm{mmHg}$ on arterial blood gas sampling or PF ratio [Horowitz index for lung function] $<300$ or lung infiltrates involving $>50 \%$ of the lung fields on a chest $x$-ray), according to the criteria designed by the World Health Organization. ${ }^{11}$

In accordance with PEMH's Institutional COVID19 Management Guidelines, the following constituted standard therapy: all patients, regardless of disease severity, received a standard protocol of aspirin, oral vitamins $\mathrm{C}$ and $\mathrm{D}$, zinc, famotidine, melatonin, antico- agulation, awake proning (if $\mathrm{PaO} 2<80 \mathrm{mmHg}$ ) and corticosteroids. All patients suffering from a Cytokine Release Storm (CRS) or hypoxemia received either methylprednisolone $1 \mathrm{mg} / \mathrm{kg}$ or dexamethasone 6-12 $\mathrm{mg} /$ day irrespective of overall disease severity. Respiratory support was given for hypoxemia and consisted of supplemental oxygen therapy, non-inva-sive ventilation (in the form of continuous positive airway pressure at $8-10 \mathrm{cmH} 2 \mathrm{O}$ in 2-4 hours sessions to maintain O2 saturation at $90-94 \%$ ) or invasive ventilation.

The patients' data was recorded in spreadsheets on Microsoft Excel and IBM SPSS version 22. The data was then analyzed using SPSS-22 and a two-sided Fisher's Exact test was used to determine whether any differences in patient outcomes between different groups were statistically significant or not. Charts were generated on Microsoft Excel.

\section{RESULTS}

The total study population was 90 male patients, aged 40-79 years (mean age: $57.28 \pm 10.33$ years), with laboratory confirmed SARS-CoV-2 infection. The age distribution of cases is shown in Table-I.

About $52(57.8 \%)$ patients did not have diabetes and $38(42.2 \%)$ patients had diabetes. Among patients without diabetes, 27 patients did not receive Therapeutic Plasma Exchange (TPE) while 25 patients did receive TPE. Among the population with diabetes, 23 subjects did not receive TPE while 15 did receive TPE (Table-II). The total number of subjects who did not

Table-I: Demographic information.

\begin{tabular}{l|c}
\hline Age Group & $\mathbf{n ~ ( \% )}$ \\
\hline $40-49$ years & $25(27.8 \%)$ \\
\hline $50-59$ years & $22(24.4 \%)$ \\
\hline $60-69$ years & $17(18.9 \%)$ \\
\hline $70-79$ years & $\mathbf{n ~ ( \% )}$ \\
\hline Table-II: Case distribution. & $15(16.7 \%)$ \\
\hline $\begin{array}{l}\text { Patient Category } \\
\text { Patients with Diabetes who received } \\
\text { Therapeutic Plasma Exchange }\end{array}$ & $23(25.6 \%)$ \\
\hline $\begin{array}{l}\text { Patients with Diabetes who did not } \\
\text { receive Therapeutic Plasma Exchange }\end{array}$ & $25(27.8 \%)$ \\
\hline $\begin{array}{l}\text { Patients without Diabetes who } \\
\text { received Therapeutic Plasma Exchange }\end{array}$ & $27(30 \%)$ \\
\hline $\begin{array}{l}\text { Patients without Diabetes who did not } \\
\text { receive Therapeutic Plasma Exchange }\end{array}$ & \multicolumn{2}{|c}{$26 \%$} \\
\hline
\end{tabular}

receive TPE was 50 . The remaining 40 subjects received 1 or more TPE sessions (Table-III).

Nine subjects ( $10 \%$ of the study population) died in the study. Of these, 6 did not receive Therapeutic 
Table-III: Therapeutic plasma exchange (TPE) sessions.

\begin{tabular}{c|c}
\hline $\begin{array}{c}\text { Number of Therapeutic } \\
\text { Plasma Exchange Sessions }\end{array}$ & $\mathbf{n ~ ( \% )}$ \\
\hline 0 & $50(55.6 \%)$ \\
\hline 1 & $12(13.3 \%)$ \\
\hline 2 & $13(14.4 \%)$ \\
\hline 3 & $9(10 \%)$ \\
\hline 4 & $5(5.6 \%)$ \\
\hline 5 & $1(1.1 \%)$ \\
\hline
\end{tabular}

Plasma Exchange (TPE). The case fatality rate in the group that did not receive TPE was $12 \%$ compared to $7.5 \%$ in the group that received 1 or more TPE sessions (Figure-1). The relative risk reduction was 37.5\%.

As shown in Figure-2, patients without diabetes who did not receive TPE had a mortality rate of $14.82 \%$ compared to $0 \%$ in patients who received TPE $(p=0.112)$. Whereas in patients with diabetes, 2 out of the 23 subjects $(8.7 \%)$ who did not receive Therapeutic Plasma Exchange died compared to 3 out of 15 subjects $(20 \%)$ who did receive TPE $(p=0.365)$.

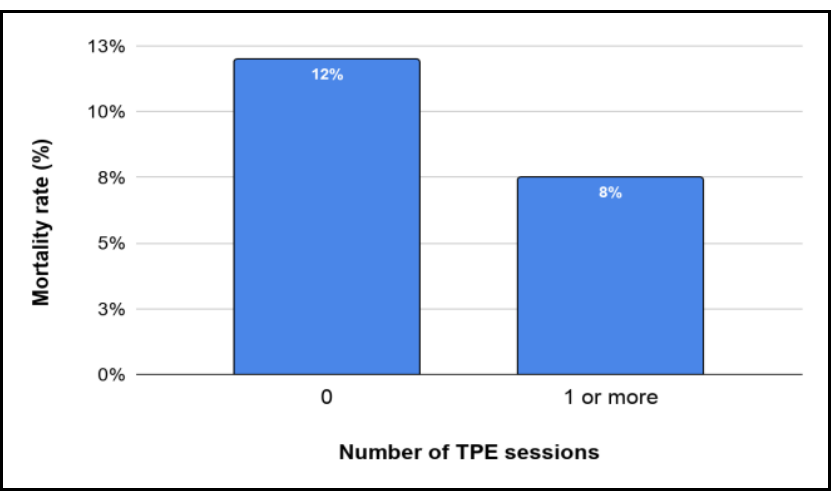

Figure-1: Number of TPE sessions and mortality rate (\%).

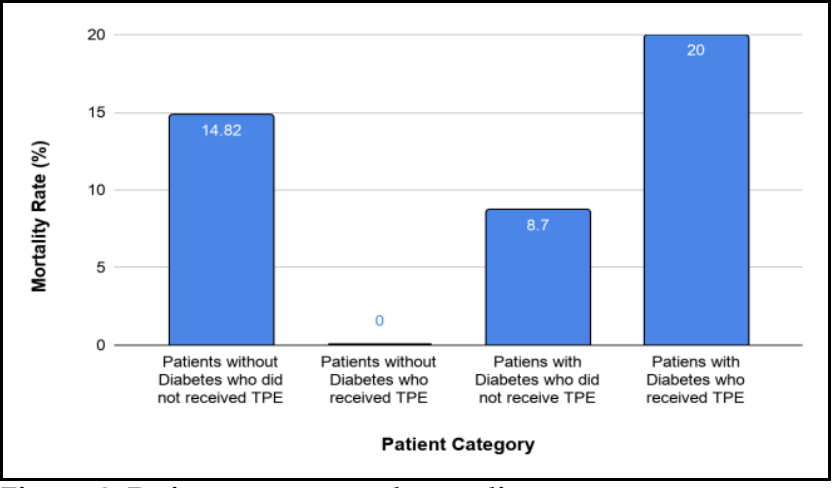

Figure-2: Patient category and mortality rate.

\section{DISCUSSION}

In our study, the mortality rate for patients who received 1 or more sessions of TPE was lower than the mortality rate for patients who received no TPE with a relative risk reduction of $37.5 \%$. Many other studies have shown a similar mortality benefit with the use of plasma exchange in COVID-19 patients. 8,12,13 In one such study, Khamis et al, reported that mortality was lower in the group on TPE compared to the group not on TPE at 14 days ( 0 versus $35 \% ; p=0.033$ ) and 28 days $\left(0\right.$ versus $35 \%$; $p=0.033$ ) following the procedure. ${ }^{12} \mathrm{Gu}-$ cyetmez et al, also reported a similar trend of decrease in mortality rate in patients who received therapeutic plasma exchange $(8.3 \%)$ as compared to the control group $(58.3 \%)(p=0.009) .{ }^{8}$

As Kamran et al, pointed out, this mortality benefit may be explained by the fact that the cytokine profile in COVID-19 patients closely resembles that of secondary hemophagocytic lymphohistiocytosis (sHLH) and is often accompanied by features of coagulopathy and septic shock; the use of therapeutic plasma exchange has been established in all of these conditions. ${ }^{9,14}$ Adeli et al, has further reiterated this point and attributed the improvement in respiratory status and reduction in pulmonary involvement of patients with COVID-19 to the elimination of cytokines. ${ }^{13}$

We found a similar trend among patients in our study who did not have diabetes where mortality in the group receiving TPE was considerably lower than the group not on TPE. However, this difference was not statistically significant ( 0 versus $14.82 \%$; $p=0.112$ ). These results are similar to a study conducted by Faqihi et al Which showed that while therapeutic plasma exchange did significantly reduce ICU length-ofstay and the need for mechanical ventilation in patients, the effect on reduction of the mortality rate was statistically insignificant $(p=0.09) \cdot{ }^{15}$

The mortality rate in patients with diabetes who received TPE was higher than in patients with diabetes who did not receive TPE. This result was found to be statistically insignificant as well $(20 \%$ vs $8.7 \%$; $p=$ 0.365). The reason for the increased mortality with TPE in COVID-19 patients who have diabetes is not clear because plasma exchange has been shown to be beneficial not only in the treatment of SARS-CoV-2 but also in reducing complications of metabolic syndrome, particularly severe hypertriglyceridemia and the micro-vascular complications associated with type 2 diabetes. ${ }^{16,17}$ In contrast to our study, Fernandez et al, demonstrated therapeutic plasma exchange as effective rescue therapy in 4 patients with multiple comorbidities, including type 2 diabetes mellitus, who had failed treatment with conventional treatment options such as antiviral agents. ${ }^{18}$

A potential reason for the statistically insignificant results reported by our study is a small sample 
size, which can lead to more type II errors and low statistical power. ${ }^{19}$ The paradoxical increase in mortality in COVID-19 patients with comorbid diabetes mellitus who received TPE as compared to patients who did not receive TPE in our study, albeit statistically insignificant, could hold clinical value and must be further looked into in future studies.

As such, we recommend that TPE should be used judiciously in patients with diabetes mellitus until further research can demonstrate therapeutic efficacy. Future studies should include larger patient populations and experimental studies should be designed to further explore the efficacy of TPE in patients with diabetes mellitus who are infected with the coronavirus.

\section{CONCLUSION}

The results of our study support the use of therapeutic plasma exchange in SARS-CoV-2 patients. There seems to be an overall reduction in mortality with the use of TPE especially in patients without diabetes mellitus. Although statistically insignificant, the mortality rate in patients with diabetes mellitus appears to be higher in those who received plasma exchange in addition to standard therapy.

\section{Conflict of Interest: None.}

\section{Authors' Contribution}

KA: Manuscript, data collection \& analysis. ZH: Article review, data collection. SMK: Manuscript review. IK: Manuscript review. ST: Manuscript, data analysis.

\section{REFERENCES}

1. Dong E, Du H. An interactive web-based dashboard to track COVID-19 in real-time. Lancet Infect Dis 2020; 20(5): 533-534.

2. World Health Organization. Weekly epidemiological update- 2 February 2021. [Internet]. Geneva: WHO; 2021, [Internet] Available from: https://www.who.int/publications/m/item/ weekly-epidemiological-update---2-february-2021 [Updated 2021 February 2; cited 2021 February 8].

3. Apicella M, Campopiano MC, Mantuano M, Mazoni L, Coppelli A, Del Prato S. COVID-19 in people with diabetes: under-standing the reasons for worse outcomes. Lancet Diabetes Endocrinol 2020; 8(9): 782-792.

4. Lim S, Bae JH, Kwon HS, Nauck MA. COVID-19 and diabetes mellitus: from pathophysiology to clinical management. Nat Rev Endocrinol 2021; 17(1): 11-30.

5. Holman N, Knighton P, Kar P, O'Keefe J, Curley M, Weaver A, et al. Risk factors for COVID-19-related mortality in people with type 1 and type 2 diabetes in England: a population-based cohort study. Lancet Diabetes Endocrinol 2020; 8(10): 823-833.
6. Yang JK, Feng Y, Yuan MY, Yuan SY, Fu HJ, Wu BY, et al. Plasma glucose levels and diabetes are independent predictors for mortality and morbidity in patients with SARS. Diabet Med 2006; 23(6): 623-628.

7. Gul MH, Htun ZM, Shaukat N, Imran M, Khan A. Potential specific therapies in COVID-19. Ther Adv Respir Dis 2020; 14(1): $1-12$.

8. Gucyetmez B, Atalan HK, Sertdemir I, Cakir U, Telci L. Therapeutic plasma exchange in patients with COVID-19 pneumonia in intensive care unit: a retrospective study. Crit Care 2020; 24(1): 492-499.

9. Kamran SM, Mirza ZE, Naseem A, Liaqat J, Fazal I, Alamgir W, et al. Therapeutic plasma exchange for coronavirus disease-2019 triggered cytokine release syndrome; a retrospective propensitymatched control study. PLoS One 2021; 16(1): e0244853.

10. Balagholi S, Dabbaghi R, Eshghi P, Mousavi SA, Heshmati F, Mohammadi S. Potential of therapeutic plasmapheresis in the treatment of COVID-19 patients: Immunopathogenesis and coagulopathy. Transfus Apher Sci 2020; 59(6): 102993.

11. World Health Organization. Clinical management of severe acute respiratory infection (SARI) when COVID-19 disease is suspected: interim guidance, 13 March 2020. Report no. WHO/ 2019-nCoV/clinical/2020.4. Geneva: WHO; 2020, [Internet] Available from: https://apps.who.int/iris/handle/10665/3 31446 [updated 2020 March 13; cited 2021 February 8].

12. Khamis F, Al-Zakwani I, Al Hashmi S, Al Dowaiki S, Al Bahrani $\mathrm{M}$, Pandak N, et al. Therapeutic plasma exchange in adults with severe COVID-19 infection. Int J Infect Dis 2020; 99(2): 214-218.

13. Adeli SH, Asghari A, Tabarraii R, Shajari R, Afshari S, Kalhor N, et al. Therapeutic plasma exchange as rescue therapy in patients with coronavirus disease 2019: a case series. Pol Arch Intern Med 2020; 130(5): 455-458.

14. Padmanabhan A, Connelly-Smith L, Aqui N, Balogun RA, Klingel R, Meyer E, et al. Guidelines on the use of therapeutic apheresis in clinical practice-evidence-based approach from the writing committee of the american society for apheresis: the eighth special issue. J Clin Apher 2019; 34(3): 171-354.

15. Faqihi F, Alharthy A, Abdulaziz S, Balhamar A, Alomari A, AlAseri $Z$, et al. Therapeutic plasma exchange in patients with life-threatening COVID-19: a randomized control clinical trial. Int J Antimicrob Agents 2021; 106334.

16. Voinov VA. therapeutic apheresis in metabolic syndrome. Immunol Endocr Metab Agents Med Chem 2018; 18(1): 38-54.

17. Wassay SAM, Dar FJ, Saleh AK, Mansoor I. Role of therapeutic plasma exchange in the treatment of severe hypertriglyceridemia: an experience. Ther Adv Endocrinol Metab 2017; 8(12): 169-172.

18. Fernandez J, Gratacos-Ginès J, Olivas $P$, Costa M, Nieto $S$, Mateo $\mathrm{D}$, et al. Plasma exchange: an effective rescue therapy in critically ill patients with coronavirus disease 2019 infection. Crit Care Med 2020; 48(12): e1350-e1355.

19. Banerjee A, Chitnis UB, Jadhav SL, Bhawalkar JS, Chaudhury S. Hypothesis testing, type I and type II errors. Ind Psy J 2009; 18(2): 127-131. 